\title{
Assessment of Cardiac Apex Kinematics Using a Real-Time 3D Magnetic Tracking System
}

\author{
E Marcelli $^{1}$, S Spolzino ${ }^{1}$, L Cercenelli ${ }^{1}$, A Cappello ${ }^{1}$, P Bagnoli ${ }^{2}$, \\ ML Costantino ${ }^{2}$, N Malagutti ${ }^{2}$, R Fumero ${ }^{2}$, G Plicchi ${ }^{1}$ \\ ${ }^{1}$ Università di Bologna, Bologna, Italy \\ ${ }^{2}$ Politecnico di Milano, Milano, Italy
}

\begin{abstract}
The assessment of Left Ventricular Apex (LVA) kinematics throughout the cardiac cycle could be useful for evaluating cardiac performance and efficiency. We proposed and evaluated in a sheep the use of a real-time $3 D$ magnetic tracking system for the analysis of LVA kinematics. LVA kinematics was assessed using a realtime $3 D$ magnetic tracking system, whose sensor was epicardially glued on the exposed LVA. Two indexes were calculated from the 3-Dimensional apex path traced by the magnetic sensor: the $3 D$ Apex Path Length (3DAPL, length of $3 D$ apex path) and the $3 D$ Apex Path Volume ( $3 D A P V$, volume containing $3 D$ apex path). Hemodynamic index of cardiac contractility (LVdP/dt $\left.t_{M A X}\right)$ was derived from Left Ventricular Pressure (LVP) measurement and evaluated against LVA kinematics parameters, at baseline and after acute ischemia, experimentally induced by coronary ligation. Results showed an opposite trend between LV hemodynamics and LVA kinematics: in the ischemic heart an increase of both $3 D A P L(+24.5 \%)$ and $3 D A P V$ $(+151.7 \%)$ occurred compared with baseline, while $L V d P / d t_{M A X}$ decreased (-36.9\%).
\end{abstract}

\section{Introduction}

Through the assessment of cardiac wall motion, it is possible to analyse cardiac kinematics, thus revealing significant information about cardiac performance and efficiency [1-6]. The displacement of the Left Ventricular (LV) walls, caused by myocardial contractions, can be considered as a composition of a translational component (both longitudinal and radial), along with a torsional movement, defined as the rotation of the Left Ventricular Apex (LVA) with respect to the LV base.

Several imaging techniques have been used to assess LV kinematics, including tagged Magnetic Resonance Imaging (tagged MRI) [1], Tissue Phase Mapping (TPM) [4], Tissue Motion Imaging (TMI) [2] and Doppler Tissue Imaging (DTI) $[3,6]$. A number of studies analysed the local contraction and torsional movements and they indicated that alterations in cardiac function influence wall kinematics $[2,3,5,6]$.

An interesting approach to evaluate cardiac performance and activity could be the assessment of cardiac kinematics not locally but globally, by analysing the displacement of the heart in 3-Dimensional (3D) space throughout the cardiac cycle. Global cardiac kinematics can be estimated by assessing the spatial displacement of a specific region of interest on the cardiac wall. In particular, the LVA could represent a significant site to focus on, as it rotates more than other cardiac regions during contraction and it is also associated with considerable translational movements.

We propose a method based on a real-time 3D magnetic tracking system, to detect and analyse the spatial movements of the LVA relatively to a static Cartesian frame of reference. A preliminary study about this new method for evaluating cardiac global kinematics is presented herein.

\section{Methods}

An adult female sheep $(60 \mathrm{Kg})$ was used for the study. The protocol was approved by the institutional animal care and use committee. All surgical procedures were performed in aseptic conditions and under general anaesthesia following a standardized protocol. The animal was positioned in a right lateral decubitus and was surgically treated to expose the heart, granting access to the LVA.

In order to acquire LV Pressure (LVP), a pressure catheter was placed in the left ventricle through the carotid artery. In order to assess LVA kinematics, a realtime magnetic tracking system (microBIRD, Ascension Technology Corporation, Burlington, VT) was used. The microBIRD system consists of a magnetic pulse transmitter unit, a small magnetic receiver sensor (1.6 $\mathrm{mm}$ diameter, $7 \mathrm{~mm}$ length) and a Control Unit (CU). Both the transmitter and the sensor contain three orthogonally-placed inductance coils, which are able to 
generate (transmitter) and detect (sensor) magnetic pulses in the three orthogonal spatial directions [Fig. 1]. The CU controls the magnetic pulse generated by the transmitter and it receives sensor-transduced signals. The CU performs real-time calculations of $\mathrm{x}, \mathrm{y}, \mathrm{z}$ coordinates of the sensor position in a global static Cartesian 3D frame of reference, by combining the data relative to the transmitted and received magnetic pulses from each coil. The CU is interfaced with an external laptop, by means of a dedicated software. For the experimental setting, the magnetic receiver sensor was inserted in a small plastic box for protection and then it was epicardially glued on the LVA [Fig. 2]. The transmitter unit was positioned at a side of the operating table, close to the sheep and facing the sensor, together with the CU connected to the laptop. The acquired $\mathrm{x}, \mathrm{y}, \mathrm{z}$ coordinates describe the spatial displacement of the LVA, defined as 3-Dimensional apex path. Two indexes are calculated from the 3-Dimensional apex path traced by the magnetic sensor: the 3D Apex Path Length (3DAPL, length of the 3D apex path) and the 3D Apex Path Volume (3DAPV, volume of the smallest cuboid containing the $3 \mathrm{D}$ apex path). 3DAPL and 3DAPV are obtained as mean values over a number of cardiac cycles:

$$
3 D A P L=\frac{\sum_{i=1}^{N-1} \sqrt{\left(x_{i+1}-x_{i}\right)^{2}+\left(y_{i+1}-y_{i}\right)^{2}+\left(z_{i+1}-z_{i}\right)^{2}}}{N c}
$$

$3 D A P V=\frac{\sum_{j=1}^{N c}\left[\max \left(x_{j i}\right)-\min \left(x_{j i}\right)\right] \cdot\left[\max \left(y_{j i}\right)-\min \left(y_{j i}\right)\right] \cdot\left[\max \left(z_{j i}\right)-\min \left(z_{j i}\right)\right]}{N c}$

where $N$ is the total number of samples, $N c$ is the total number of cardiac cycles, $j$ is the cycle counter and $i$ is the sample counter.

Tracking data about LVA position, provided by the microBIRD system, were acquired and processed by using an ad-hoc acquisition software, which was developed by our team (using LabVIEW 8.2, National Instruments, Austin, TX, USA). LVP and ECG were also continuously recorded using the same acquisition software, thus obtaining hemodynamic information and synchronism with heart cycles.

Starting from baseline condition, an impairment of cardiac function was experimentally induced in the animal: acute ischemia was obtained by ligation of the left anterior descending coronary artery, which was maintained for 2 minutes and subsequently released. 3Dimensional apex path, LVP and ECG were continuously recorded. The positive peak of the first derivative of LVP $\left(\mathrm{LVdP} / \mathrm{dt}_{\mathrm{MAX}}\right)$ was calculated to monitor the alteration of cardiac function.

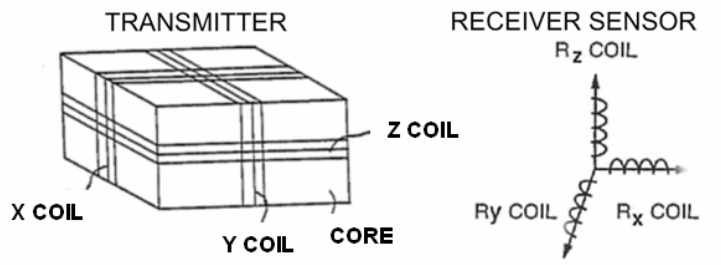

Figure 1. Schemes of coils positioning in the magnetic transmitter (left) and in the receiver sensor (right) of the microBIRD system.

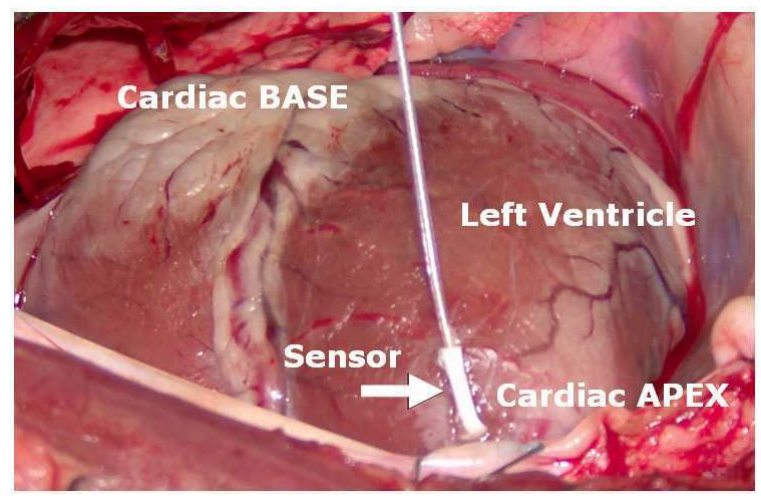

Figure 2. View of the exposed Left Ventricular Apex (LVA) with the magnetic sensor epicardially fixed (arrow).

\section{Results}

The 3D apex path curve assessed during baseline displayed a typical closed-loop shape, which repeated itself every cardiac cycle without significant variations. In Figure 3 we report an example of the acquired 3D apex path curve, which is shown during various phases of the cardiac cycle: from $\mathrm{R}$ wave to $\mathrm{T}$ wave onset $(\mathrm{A})$; from $\mathrm{T}$ wave onset to $\mathrm{T}$ wave end (B); from $\mathrm{T}$ wave end to next $\mathrm{R}$ wave (C). At the end of systole, the LVA changes direction due to myocardial diastolic relaxation, as it can be observed in the 3D apex path segment associated to phase B. However, the LVA does not trace the same path backwards and does not return to its original position due to hysteresis of the cardiac wall fibers and changes in blood flow. Moreover, some overshooting is observed during diastole $(\mathrm{C})$ and the LVA returns approximately to its initial position only at end of the cycle, thanks to the elastic recall of the heart wall. At bottom of Figure 4 graph of 3DAP acquired during 3 subsequent cardiac cycles. 


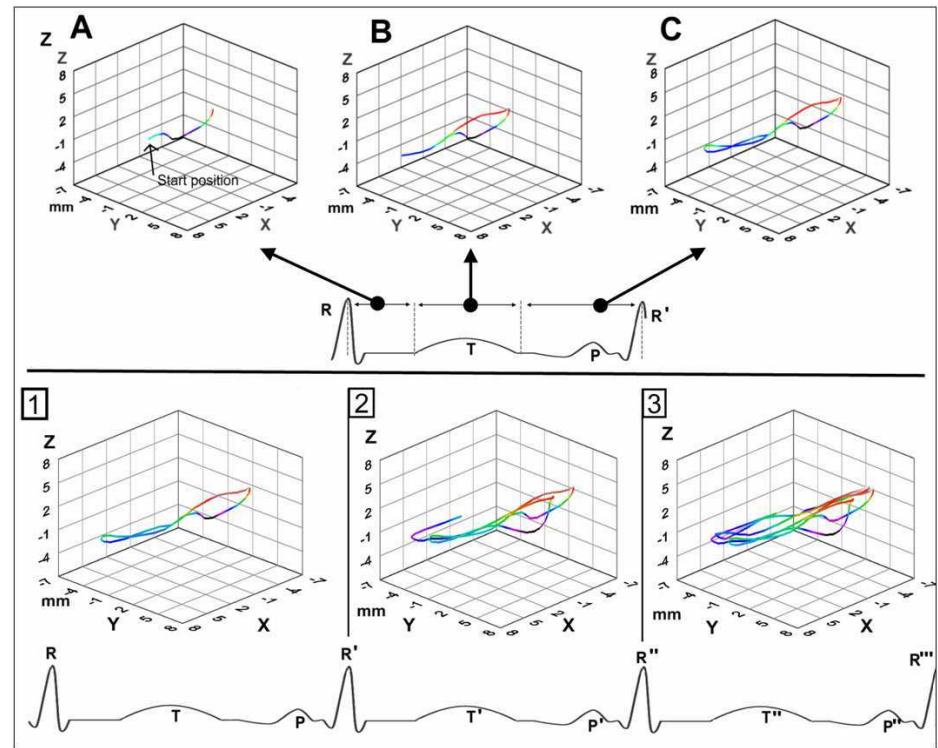

Figure 3. (top): 3-Dimensional apex path curve ( $\mathrm{mm}$ ) assessed throughout one cardiac cycle; each graph refers to a specific phase of the cardiac cycle, as shown on the ECG waveform below; (bottom): 3-Dimensional apex path curve during 3 subsequent cardiac cycles at baseline condition. Colour coding: $Z$ coordinate values (dark purple=low, light red=high).

During experimentally induced ischemia (two sequences of coronary ligation - release of ligation) the trend of the $\mathrm{LVdP} / \mathrm{dt}_{\mathrm{MAX}}$ curve was opposite to those of both 3DAPL and 3DAPV [Fig. 4]: a decrease of $\mathrm{LVdP} / \mathrm{dt}_{\mathrm{MAX}}$ values (from basal value of $876 \pm 3 \mathrm{mmHg} / \mathrm{s}$ to the minimum value of $552 \pm 10 \mathrm{mmHg} / \mathrm{s}$ ) occurred, while a slight increase of 3DAPL (from basal value of $33.0 \pm 1.0 \mathrm{~mm}$ to the maximum value of $41.1 \pm 0.7 \mathrm{~mm}$ ) and a significant increase of 3DAPV (from basal value of $4319 \pm 281 \mathrm{~mm}^{3}$ to the maximum value of $10872 \pm 468$ $\mathrm{mm}^{3}$ ) were observed. This can also be observed by considering the 3D apex path curve acquired over a cardiac cycle [Fig. 5]: 3DAPL and 3DAPV are significantly greater during coronary ligation than at baseline. At the time of release of ligation both 3DAPL and 3DAPV values continued to increase rapidly (maximum values: $45 \pm 1 \mathrm{~mm}$ and $16326 \pm 467 \mathrm{~mm}^{3}$, respectively) for a short period (60 cardiac cycles), then suddenly dropped back to basal values.

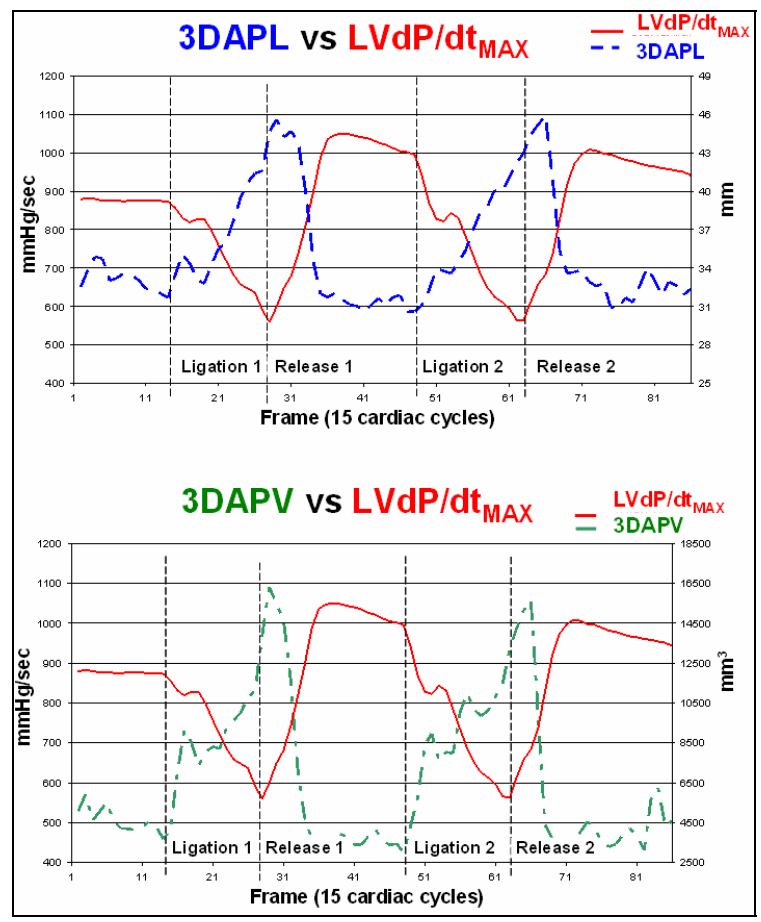

Figure 4. Trend of $\mathrm{LVdP} / \mathrm{dt}_{\mathrm{MAX}}$ (solid red line), 3DAPL (dashed blu line, top graph) and 3DAPV (dashed green line, bottom graph) during two coronary ligations (and release). The reported values are means over 15 cardiac cycles. 


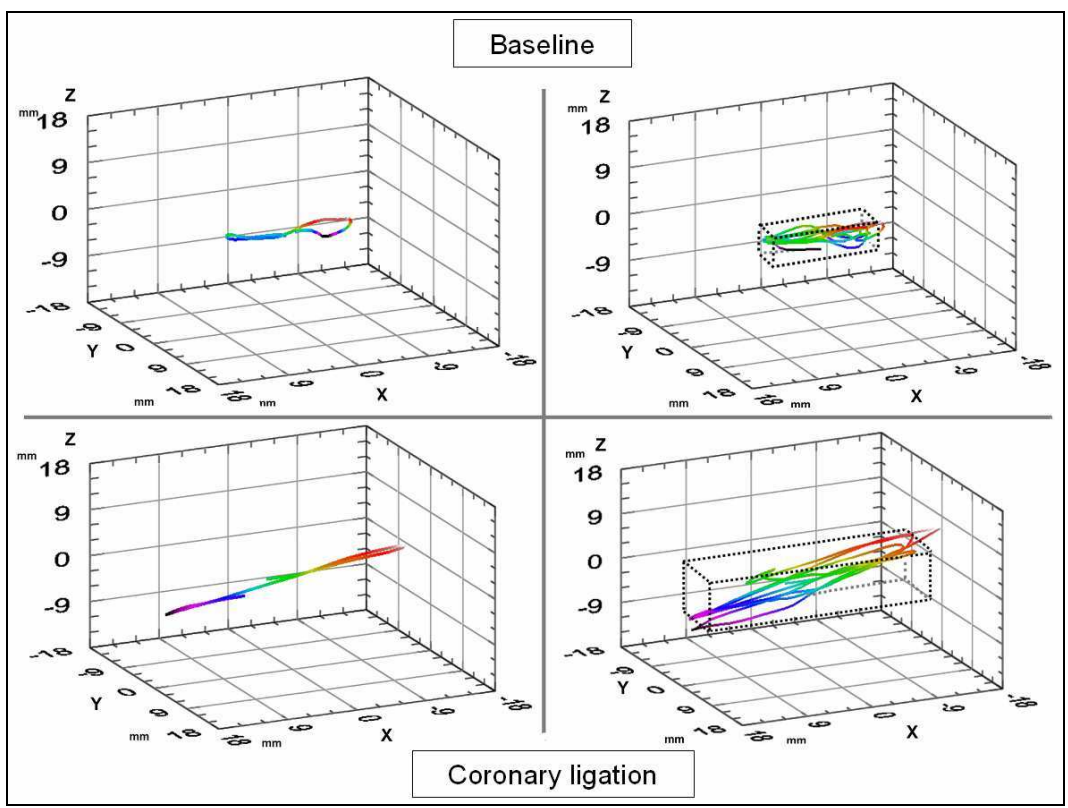

Figure 5. 3D apex path curves $(\mathrm{mm})$ acquired at baseline (top) and during acute ischemia induced by coronary ligation (bottom), during 1 (left) and 5 subsequent cardiac cycles (right); in dotted lines graphical schematization of the 3DAPV cuboid.

\section{Discussion and conclusions}

Data showed an existing link between cardiac energy efficiency and LVA kinematics. In the impaired heart LVA displacement over a number of cardiac cycles appeared to be greater than under normal conditions, exhibiting a correlation between apex dyskinesia caused by abnormal apex movements and a decreasing trend of $\mathrm{LVdP} / \mathrm{dt}_{\mathrm{MAX}}$. Myocardial ischemia induced by coronary ligation provokes an acute reduction of oxygen supply to myocardial regions. This determines an altered contraction mechanism and apex dyskinesia, reflected by the increase of 3DAPL and 3DAPV values. The disordered cardiac movements, however, seem to waste energy, as showed by the drop in the cardiac haemodynamic function ( $\left.\mathrm{LVdP} / \mathrm{dt}_{\mathrm{MAX}}\right)$. At the moment of coronary artery release, the increase in 3DAPL and 3DAPV values could be interpreted as an early reaction of the heart in an attempt to minimise the recovery time from the previous loss of cardiac efficiency.

One of the major drawbacks of the proposed method is that the $3 \mathrm{D}$ magnetic tracking system is intrinsically sensitive to electrical noise and signal distortion induced by ferromagnetic metals. Thus, preliminary preparation of the experimental setting (i.e. removing all unnecessary electronic or ferromagnetic equipment) was necessary in order to minimize electromagnetic interference and to obtain accurate measurements. Further investigation is required to better understand the value of $3 \mathrm{D}$ apex path assessment and its potentiality in clinical use. The opportunity of developing an implantable catheter-based tracking sensor should be explored, in order to provide a system for chronic cardiac function monitoring based on
LVA kinematics assessment.

\section{References}

[1] Moore CC et al.. Three-dimensional systolic strain patterns in the normal human left ventricle: characterization with tagged MR imaging. Radiology 2000; 214(2): 453-66.

[2] Storaa $\mathrm{C}$ et al.. Tissue motion imaging of the left ventricle-quantification of myocardial strain, velocity, acceleration and displacement in a single image. Eur. J Echocardiography 2004; 5(5): 375-385.

[3] Notomi $\mathrm{Y}$ et al.. Enhanced ventricular untwisting during exercise: a mechanistic manifestation of elastic recoil described by Doppler tissue imaging. Circulation 2006; 113(21): 2524-2533.

[4] Jung $\mathrm{B}$ et al.. Investigating myocardial motion by MRI using tissue phase mapping. Eur J of Cardiothorac Surgery 2006; 29(Suppl 1): S150-7.

[5] Fuchs E et al.. Cardiac rotation and relaxation in patients with chronic heart failure. Eur J Heart Fail 2004; 6(6): 71522.

[6] Miyatake $\mathrm{K}$ et al.. New method for evaluating left ventricular wall motion by color-coded tissue Doppler imaging: in vitro and in vivo studies. J Am Coll Cardiol 1995; 25(3): 717-24.

Address for correspondence:

Emanuela Marcelli

Università di Bologna

Dipartimento Clinico di Scienze Radiologiche e

Istocitopatologiche - Sezione Tecnologie Biomediche

Policlinico S. Orsola Malpighi

Via Massarenti, 9 (Palazzina 17)

40138 Bologna, Italy

emanuela.marcelli@unibo.it 